\title{
A Cantilever-type Electrostatic Zipping Actuator
}

\author{
Naresh Dhaubanjar ${ }^{1,2}$, Smitha M. N. Rao ${ }^{1}$, Ying Cai ${ }^{2}$, Dan Popa ${ }^{1}$, \\ $\mathrm{Mu} \mathrm{Chiao}{ }^{3}$ and J.-C. Chiao ${ }^{1,2}$ \\ 1. Automation \& Robotics Research Institute, 2. Department of Electrical Engineering, \\ The University of Texas at Arlington, USA \\ 3. Department of Mechanical Engineering, The University of British Columbia, Canada
}

\begin{abstract}
This paper discussed modeling, design, fabrication and characterization of a new cantilever-type electrostatic zipping actuator. The actuator was designed to achieve high displacements and fabricated using multi-layer polysilicon foundry fabrication process PolyMUMPS. The high out-of-plane displacement is to satisfy the requirements in specific optical applications. In this paper we presented the design considerations in displacement, electrostatic forces and electrostatic stability. The electrostatic force between the curved cantilever and the bottom electrode on the substrate pulls the cantilever down. With a warped cantilever, the force closes the gap from the anchor end and gradually the zipping effect actuates the entire cantilever without increasing the biasing voltages. Previous electrostatic zipper actuators require a thin layer of dielectric material on top of the bottom electrode to prevent electrical shorting. They may have an issue with electrical breakdown of the thin dielectric layer due to the film quality. We designed a new mechanical structure to avoid the electrical shorting problem without a layer of dielectric material. Our analysis and experimental results demonstrated that the proposed design can withstand high voltages without shorting and is capable of high deflection. The vertical displacements of different device configurations were found ranging from $30.4 \mu \mathrm{m}$ to $450 \mu \mathrm{m}$ while the actuation voltages varied in the range from $12 \mathrm{~V}$ to $45.3 \mathrm{~V}$ for complete actuation. The pull-in voltages for various configurations were analyzed and presented.
\end{abstract}

Key words: Zipper actuator, electrostatic actuator, pull-in voltage, RF MEMS.

\section{INTRODUCTION}

The fields of MEMS (micro-electro-mechanical systems) and optical microsystems are at a fast growing stage. Optical MEMS has become a promising technology in the fields of telecommunication, high-resolution display, and medical imaging. Various actuation mechanisms have been used to actuate micro optical devices such as electromagnetic, electrostatic, thermal, and piezoelectric actuation [1-4]. Electrostatic actuators show advantages of low power consumption, high actuation speeds, and fabrication compatibility [5]. Both large deflection and high force can be achieved by using the cantilever type electrostatic zipping actuator. Electrostatic actuators with an S-shaped film element and deformed membrane have been reported with high displacements [6,7]. Legtenberg et al. have demonstrated laterally compliant curved electrode zipping actuators [8]. Curved electrode has been used in microactuators to align optical fibers [9]. A large gap between the top and the bottom electrodes increases the displacement yet also the voltage required to actuate the actuator. The zipping actuator works better if the gap between the electrodes is small at the beginning of zipping action [10].

The zipper actuator can be fabricated using various fabrication processes such as SOI (silicon on insulator), bulk micromachining and surface micromachining. They can be done monolithically using conventional semiconductor processes. Therefore, they could be cost effective in mass production.

The actuation principle of the zipper actuator is based on the electrostatic force between a curved cantilever and a bottom electrode that pulls the cantilever down. Previous electrostatic zipper actuators require a thin layer of dielectric material such as oxide or nitride on top of the bottom electrode to prevent electrical shorting. To circumvent the problem of electrical shorting and dielectric breakdown, we proposed an improved design for a surface micromachined cantilever-type zipper actuator. This actuator can be utilized in optical applications for switching, scanning and power regulation. The analysis and experimental results demonstrated that our design can withstand high voltages without

Smart Structures, Devices, and Systems III, edited by Said F. AI-Sarawi, Proc. of SPIE Vol. 6414, 641421, (2007) · 0277-786X/07/\$15 - doi: 10.1117/12.695959 
shorting and is capable of high deflections. The zipper actuator was characterized statically and dynamically. In this paper, the design consideration in displacement, electrostatic force, and electrostatic stability of the zipper actuator is presented. The finite element analysis is used to verify the static and dynamic results.

\section{ACTUATOR DESIGN}

The mask layouts and a photo of the actuator after hydrofluoric (HF) release of the curved cantilever-type electrostatic zipper actuator are shown in Fig. 1 (a), (b), and (c), respectively. After the HF release process, the top electrode curves up due to the bending stress and thermal expansion of the polysilicon and metal layers. When the voltage is applied on the contact pad, the electrostatic force between the curved cantilever and the bottom electrode on the substrate pulls the cantilever down. With a warped cantilever, the force closes the gap from the anchor end and gradually the zipping effect actuates the entire cantilever without increasing the biasing voltages. The mechanical structure shown in Fig. 1 prevents the top and bottom electrodes from contacting each other, thus keeping the device from being short- circuited.

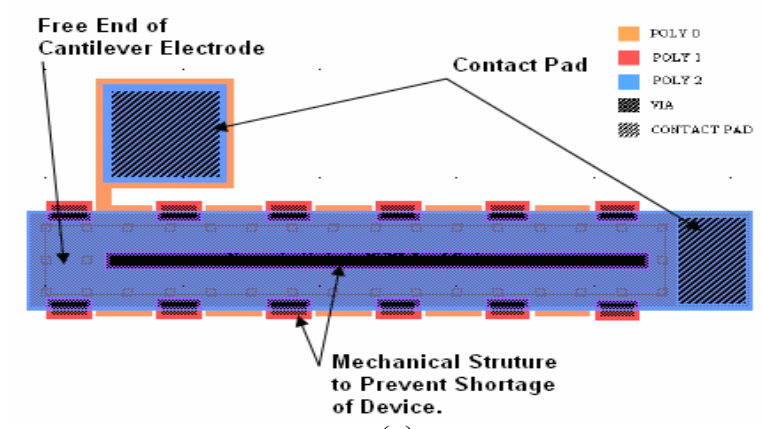

(a)

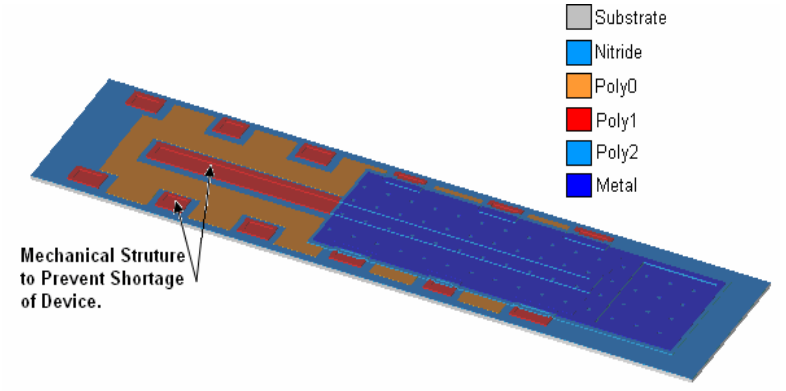

(b)

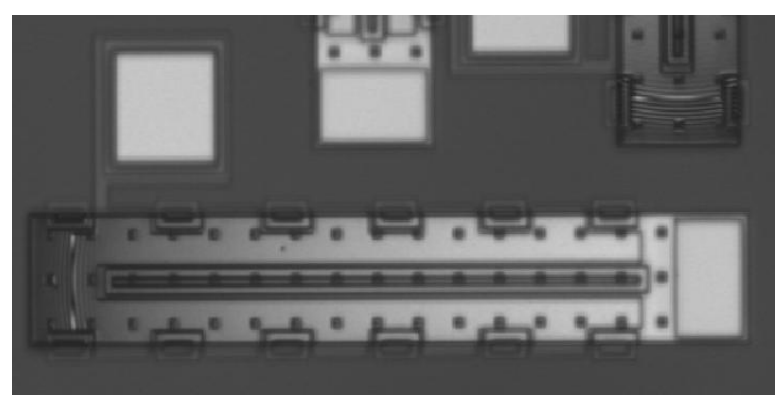

(c)

Figure 1: Cantilever type electrostatic zipper actuator. (a) The mask layout. (b) Detail view of the mechanical structure. (c) A photo of the device after HF release.

\section{LUMPED MODELING ANALYSIS}

\subsection{Stress induced bending}

Micromachined cantilever beams are subject to residual stresses due to the fabrication process $[11,12]$. This stress causes the cantilever beam to curl upwards. During the HF release process, the chips are heated, and the cantilever beams deflect fully downwards touching the substrate due to the thermal expansion of metal. Upon returning to the room temperature, the cantilevers curve up more than in the stress-induced state. The schematic of the cantilever beam is shown in Fig. 2 with thickness' of $h_{1}$ and $h_{2}$, residual stress of $\sigma_{1}$ and $\sigma_{2}$, and Young's modulus of $E_{1}$ and $E_{2}$ for polysilicon and gold layers, respectively. The force and moments acting on the structure are also shown in the figure [13]. The radius of curvature of the cantilever solely due to the residual stress is given by 


$$
\rho=\frac{\left(w_{1} E_{1} h_{1}{ }^{2}\right)^{2}+\left(w_{2} E_{2} h_{2}{ }^{2}\right)^{2}+2 w_{1} w_{2} E_{1} E_{2} h_{1} h_{2}\left(2 h_{1}^{2}+3 h_{1} h_{2}+2 h_{2}^{2}\right)}{6 w_{1} w_{2} E_{1} E_{2} h_{1} h_{2}\left(h_{1}+h_{2}\right)\left(\frac{\sigma_{2}}{E_{2}}-\frac{\sigma_{1}}{E_{1}}\right)}
$$

And the total deflection at the tip of the free end is

$$
d=\rho\left(1-\operatorname{Cos}\left(\frac{L}{\rho}\right)\right)
$$

where $L$ is length of the cantilever beam.
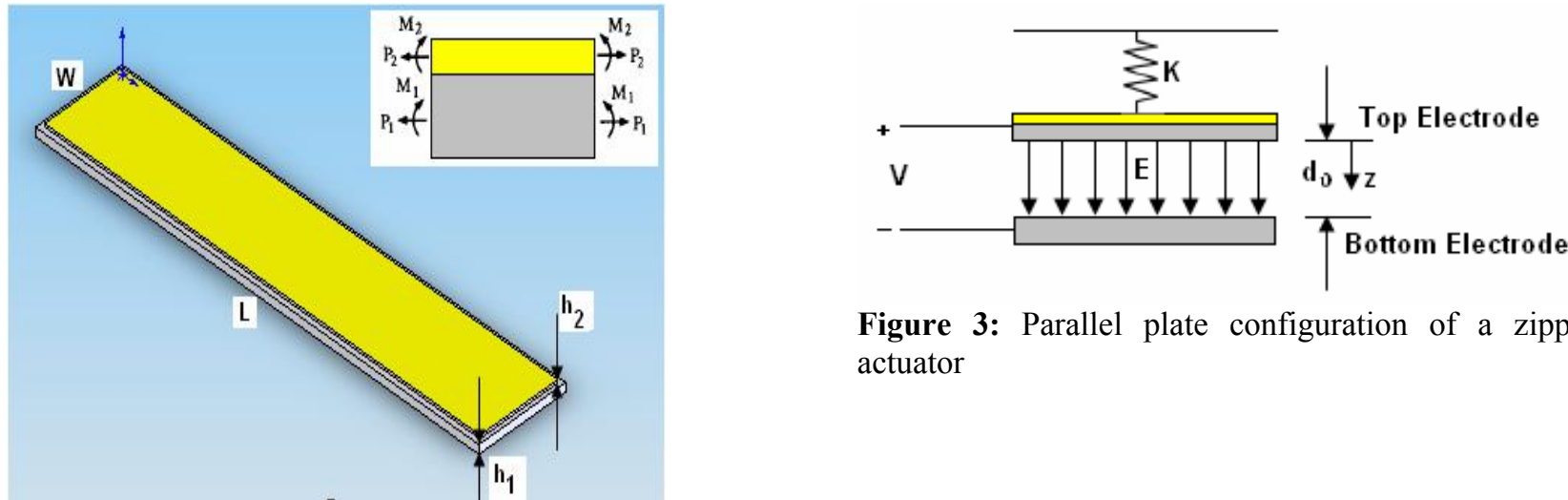

Figure 3: Parallel plate configuration of a zipper actuator

Figure 2: Schematic of the cantilever beam.

The radius of curvature due to the residual stress and curing temperature is given by

$$
\rho=\frac{\left(w_{1} E_{1} h_{1}{ }^{2}\right)^{2}+\left(w_{2} E_{2} h_{2}^{2}\right)^{2}+2 w_{1} w_{2} E_{1} E_{2} h_{1} h_{2}\left(2 h_{1}{ }^{2}+3 h_{1} h_{2}+2 h_{2}{ }^{2}\right)}{6 w_{1} w_{2} E_{1} E_{2} h_{1} h_{2}\left(h_{1}+h_{2}\right)\left(\alpha_{2}-\alpha_{1}\right) \Delta T}
$$

where, $w_{1}$ and $w_{2}$ are the width of polysilicon and gold layers, respectively; $\Delta T$ is the difference between the curing temperature and the room temperature; $\alpha_{1}$ and $\alpha_{2}$ are thermal expansion coefficients of polysilicon and gold layers, respectively.

The total deflection of the cantilever can be calculated using Eq.2, and a MatLAB code to solve for the total stress induced deflection and the deflection due to the residual stress and curing temperature. For a cantilever electrode of $475-\mu \mathrm{m}$ long and $90-\mu \mathrm{m}$ wide, the total deflection, solely due to stress, is $16.285 \mu \mathrm{m}$. In our calculations, the induced stress due to fabrication processes was given by the PolyMUMPS database as $7.8 \mathrm{MPa}$ for polysilicon and 23.3MPa for gold. Similarly, the deflection due to the residual stress and change in temperature is found to be $34.334 \mu \mathrm{m}$ at a curing temperature of $100^{\circ} \mathrm{C}$ and the room temperature of $30^{\circ} \mathrm{C}$.

\subsection{Electrostatic force}

A general analysis for the electrostatic force of the cantilever type zipper actuator can be performed by considering a small section of a parallel plate capacitor configuration composed of a moveable plate of a mass $M$ and a spring constant $K$ separated from the bottom electrode by an air gap $d_{o}$ [14]. The configuration is shown in Fig. 3. A constant voltage is applied between the top and bottom electrodes. Electrical energy is stored in the electric fields established by the charges accumulated in the capacitor due to the applied electric potential. The electrostatic force pulls the top electrode towards the bottom electrode causing the top electrode to deflect. This electrostatic force is inversely 
proportional to the air gap between the top and bottom electrodes. For lumped modeling analysis, damping within the system was neglected. The electrostatic force as a function of bias voltage is

$$
F_{\text {electrostatic }}=\frac{\varepsilon_{0} A V^{2}}{2\left(d_{0}-z\right)^{2}}
$$

where $A$ is the area of moveable plate, $\varepsilon_{0}$ is the permittivity of free space, $d_{o}$ is the initial gap between the top and bottom electrodes, $z$ is the displacement, and $V$ is the applied potential.

\subsection{Pull-in voltage}

The pull-in action is related to the elastic and electrostatic forces. When electric potential is applied across the two electrodes, the electrostatic force generated causes the top electrode to move towards the bottom electrode. The elastic force is a linear function of the top electrode displacement. In contrary, the electrostatic force is inversely proportional to the air gap between the two electrodes. At a certain voltage, the electrostatic force becomes larger than the elastic force when the system can not reach a force balance causing the top electrode to collapse onto the bottom electrode. This phenomenon is called pull-in. Chowdhury et al. [15] have formulated a closed-form expression for the pull-in voltage:

$$
\begin{aligned}
& V_{P I}=\sqrt{\frac{2 \widetilde{E} h^{3} d_{0}}{8.37 \varepsilon_{0} l^{4}\left(\frac{5}{6 d_{0}{ }^{2}}+\frac{0.19}{d_{0}{ }^{1.25} w^{0.75}}+\frac{0.19}{d_{0}{ }^{1.25} l^{0.75}}+\frac{0.4 h^{0.5}}{d_{0}{ }^{1.5} w}\right)}} \\
& \widetilde{E}=\frac{E}{1-v^{2}}
\end{aligned}
$$

where $E$ is the Young's modulus and $v$ is the Poisson ratio.

This formulation assumes that the top electrode has only one layer of material. The top electrode of a zipper actuator in our design has two layers, $0.5-\mu \mathrm{m}$ thick gold layer deposited on top of a $1.5-\mu \mathrm{m}$ thick polysilicon layer, with Young's modulus 168GPa and 77.2GPa for polysilicon and gold, respectively [16]. Assuming the Young's modulus of the bilayer cantilever is likely between $77.2 \mathrm{GPa}$ and $168 \mathrm{Gpa}$, the estimated pull-in voltage for the cantilever actuator with a length of $475 \mu \mathrm{m}$ and a width of $90 \mu \mathrm{m}$ should be in the range of $4.5 \mathrm{~V}$ to $6.7 \mathrm{~V}$.

\section{EXPERIMENTAL RESULTS}

Zipper actuator with various lengths and widths were fabricated using the MUMPs process run \#71. The MUMPs chips were released using the HF release process and the sequential curing procedure. The scanning electron micrograph (SEM) images of the various configurations are shown in Fig. 4.
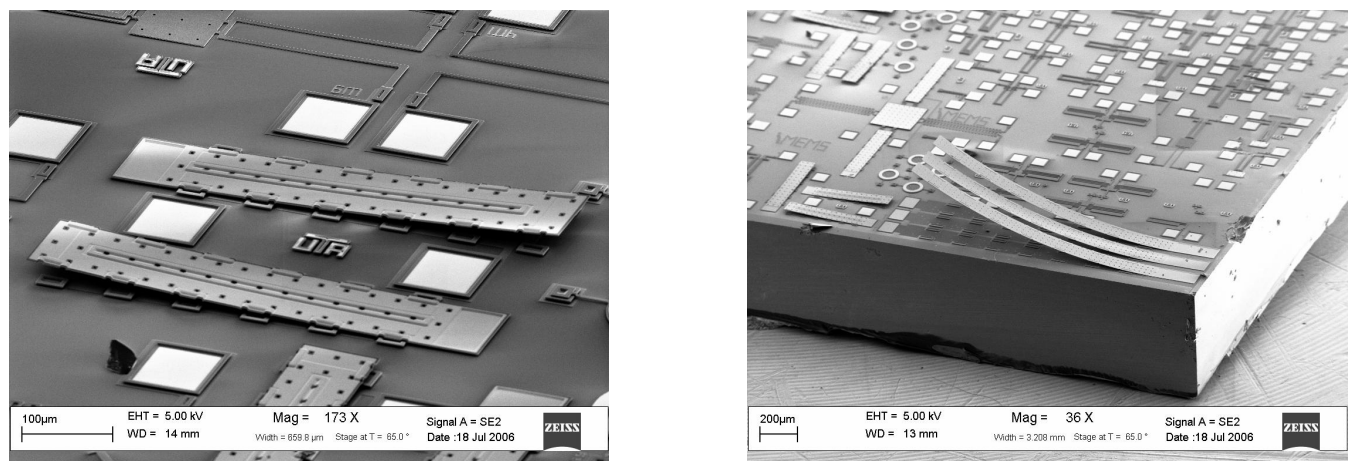

Figure 4: SEM images of various configurations of zipper actuators. 
The maximum tip deflection of the actuator was determined experimentally using an optical profiler and a reflective microscope. The optical profiler, VEECO WYKO NT1100, uses an interferometric microscope to measure the vertical displacement of the actuator. The experimental setup using the optical profiler to measure the deflection is shown in Fig. 5. The actuation voltage was provided by a voltage amplifier through sharp nickel probe tips on the contact pads. The actuators were statically and dynamically analyzed. The actuator tip deflections were measured with an input voltage varied from $0 \mathrm{~V}$ to $45 \mathrm{~V}$ in a step of $0.2 \mathrm{~V}$. The static displacement of the actuator is shown in Fig. 6 . The height of the cantilever decreases slowly as the voltage increases until 7.8 V. The cantilever electrode then collapses to the bottom electrode at $7.8 \mathrm{~V}$. The deflection of the electrode at this point could not be controlled even at a voltage increment of $0.05 \mathrm{~V}$. The pull-in voltage of $7.8 \mathrm{~V}$ is verified in Fig. 6 with a finer voltage increment. The discrepancy between the theoretical and experimental pull-in voltages might be due to the finite resistance of the nickel probe tip. The voltage applied on the contact pads was reduced due to the potential drop across the probe.

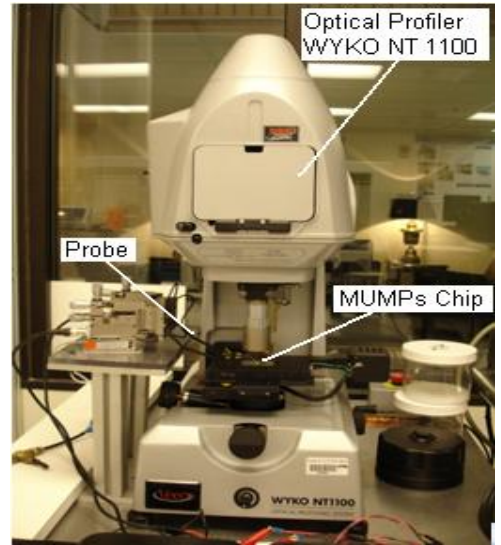

Figure 5 Experimental setup to measure deflection of actuator using an optical profiler.

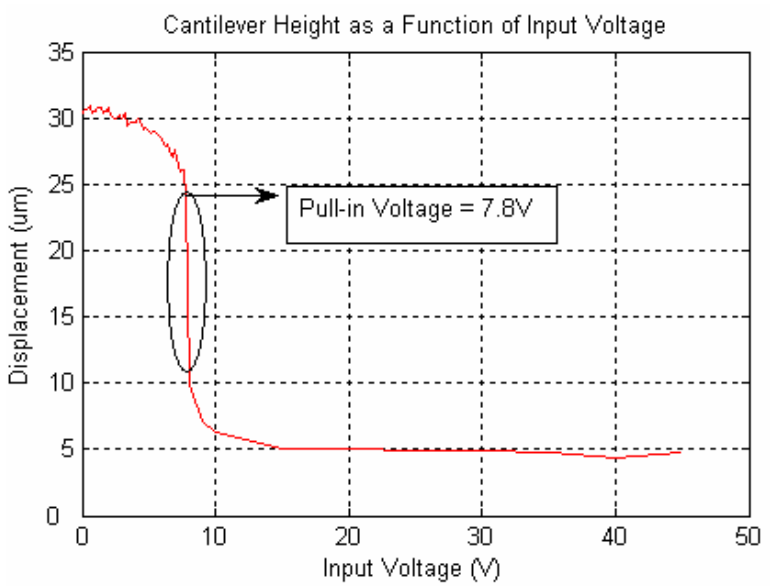

Figure 6 Pull-in voltage of the zipper actuator.

Fig. 6 shows the maximum tip deflection of cantilever is $30.4 \mu \mathrm{m}$ and the theoretical estimation is $34.33 \mu \mathrm{m}$. In the next section, the finite element simulation prediction is $35.58 \mu \mathrm{m}$. Fig. 7 shows the profiles of the actuator height along the length and tip of the cantilever, respectively. A 3-D profile of the zipper actuator obtained from optical profiler is shown in Fig 8.
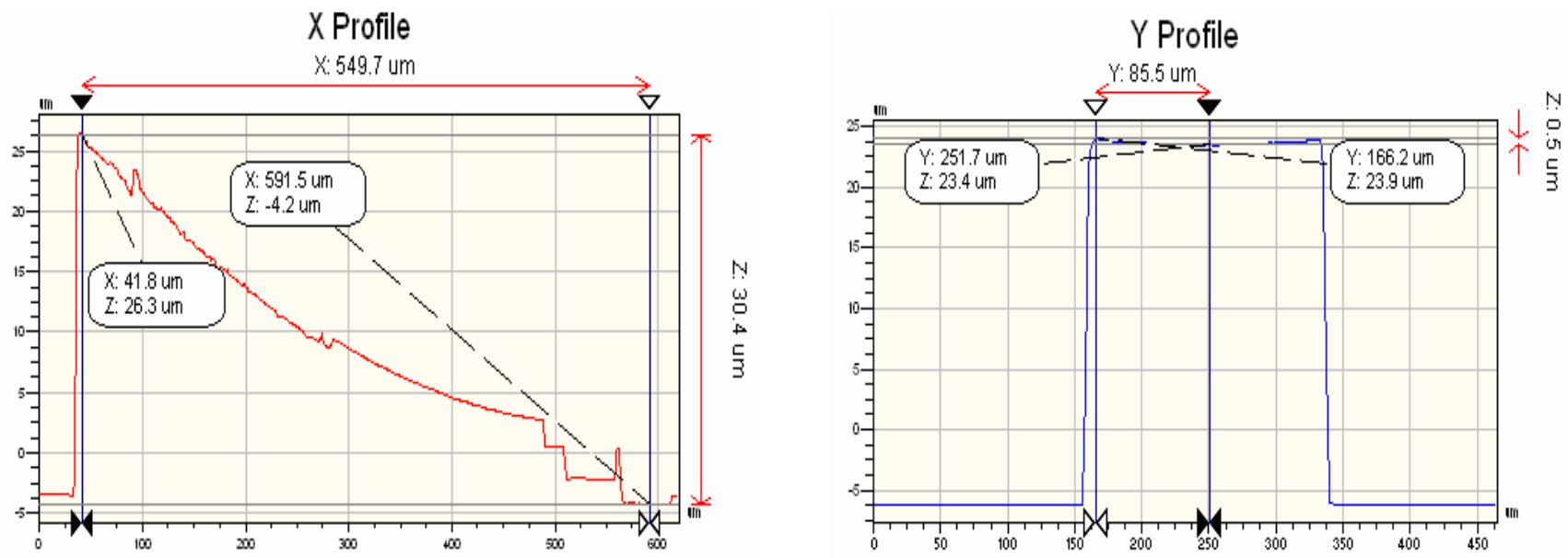

Figure 7 Profiles of the actuator height along the length and tip of the cantilever 


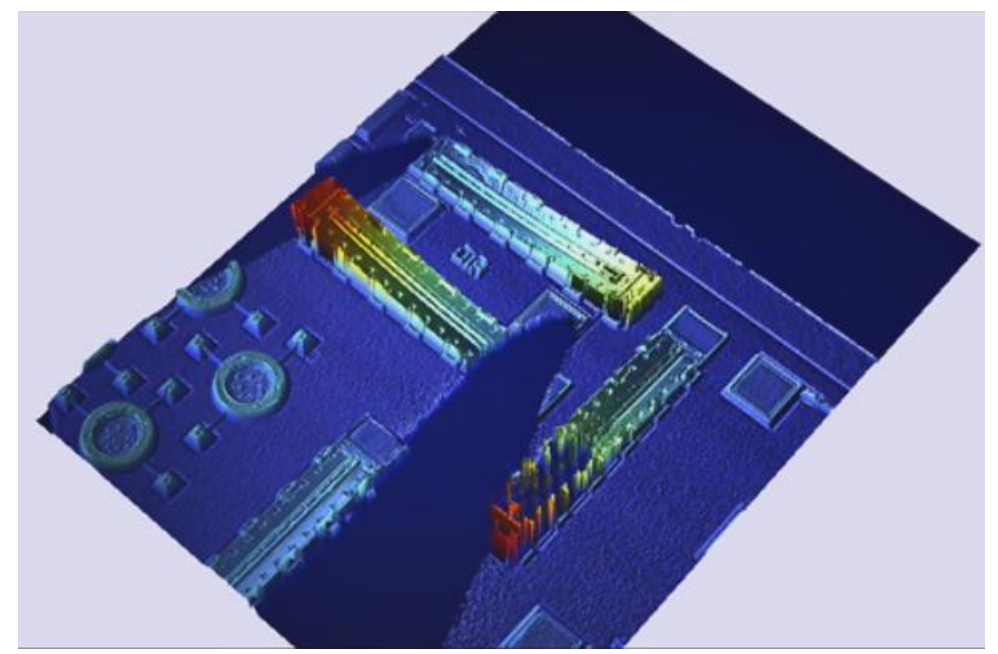

Figure 8 A 3-D profile of the zipper actuator.

\section{FINITE ELEMENT ANALYSIS}

Finite element analysis was used to validate the theoretical and experimental results. ANSYS and FemLAB have been used for simulation. A three dimensional model was built in Solidworks and then imported to ANSYS and FemLAB. The residual stress is assumed to be uniformly distributed across the top electrode surface.

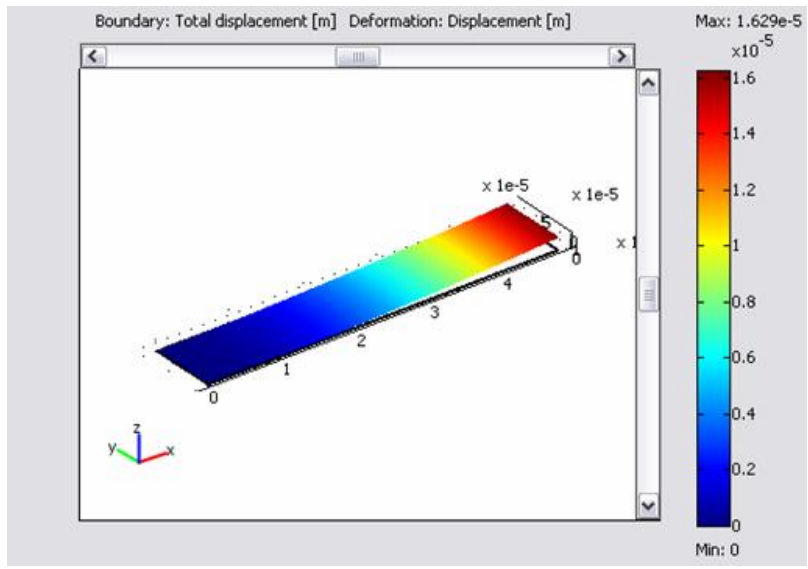

Figure 9 Stress induced deflection of a zipper actuator.

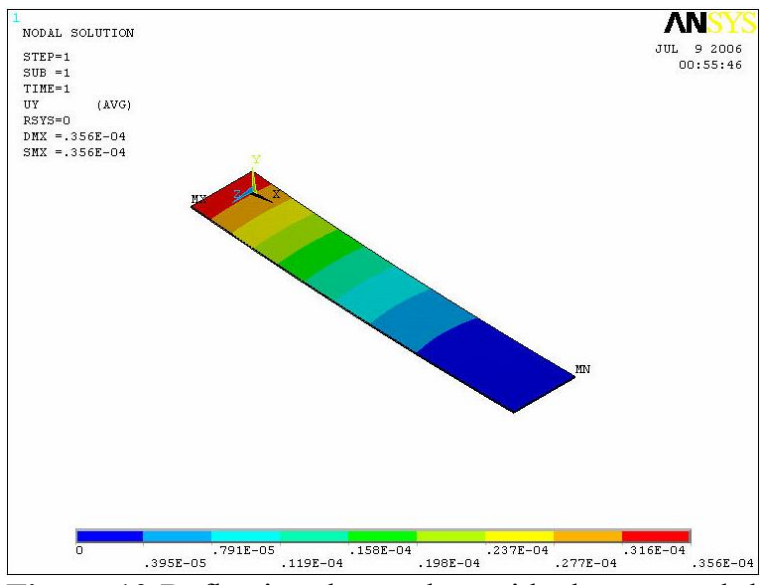

Figure 10 Deflection due to the residual stress and the curing temperature.

The "Solid, stress-strain" available in the MEMS module is used in the FemLAB simulation for the stress induced deformation. The simulation result is shown in Fig 9. The total deflection obtained from simulation is $16.29 \mu \mathrm{m}$ which is in good agreement with the theoretical value of $16.285 \mu \mathrm{m}$ by the lumped element model. For the deflection due to the residual stress and change in temperature, the cantilever structure is meshed with a solid element called "Solid186". A sequential simulation is performed where the beam is first subjected to the stress. The deformed structure thus obtained is heated to $100^{\circ} \mathrm{C}$ and then brought back to the room temperature. The simulation result is shown in Fig 10 .

The total deflection due to the residual stress and change in temperature obtained from simulation is $35.58 \mu \mathrm{m}$ which is in good agreement with the theoretical value of $34.33 \mu \mathrm{m}$. 
The material properties used for the finite element simulation is tabulated in Table 1.

Table 1 Material properties of poly-silicon and gold.

\begin{tabular}{|l|c|c|}
\hline Material Properties & Poly-Silicon & Gold \\
\hline Young's Modulus $(\mathrm{GPa})$ & 168 & 78 \\
\hline Poisson's Ratio & 0.22 & 0.42 \\
\hline Density $\left(\mathrm{kg} / \mathrm{m}^{3}\right)$ & 2329 & 19320 \\
\hline Thermal Expansion Coefficient $\left({ }^{\circ} \mathrm{C}\right)$ & $3.5 \times 10^{-6}$ & $14.2 \times 10^{-6}$ \\
\hline Thermal Conductivity $\left(\mathrm{pW} / \mu \mathrm{m}^{\circ} \mathrm{C}\right)$ & $150 \times 10^{6}$ & $350 \times 10^{6}$ \\
\hline Residual Stress $(\mathrm{MPa})$ & -7.3 & 23.6 \\
\hline
\end{tabular}

\section{CONCLUSIONS}

In this paper, a cantilever-type electrostatic zipper actuator was presented. The device was fabricated using multilayer polysilicon process, PolyMUMPS. The static and dynamic characterization was performed. The actuator was characterized in terms of electrostatic force, pull-in voltage and deflection. A mechanical structure was designed to prevent the electrical shorting of the device without the use of insulation dielectric material. The theoretical and experimental results were verified using the finite element analysis. The actuator can be utilized in optical applications to steer optical beams for switching and scanning.

\section{ACKNOWLEDGEMENT}

This research is sponsored by the National Science Foundation (NSF) DMI \#0428884. The authors would like to express their gratitude to the NSF support.

\section{REFERENCES}

1. L. K. Lagorece, O. Brand, and M. G. Allen, "Magnetic microactuators based on polymer magnets," Journal of Microelectromechanical System, 8: 2-9, 1999.

2. K. F. Harsh, B. Su, W. Zhang, V. M. Bright, and Y. C. Lee, "The realization and design considerations of a flipchip integrated MEMS tunable capacitor," Sensors and Actuators, A 80:108-118, 2000.

3. D. Yan, A. Khajepour, and R. Mansour, "Design and modeling of MEMS bidirectional vertical thermal actuator," Journal of Micromechanics and Microengineering, 14:841-850, May 2004.

4. Masayuki Kurita, Takanori Yamazaki, Hidekazu Kohira, Masaaki Matsumoto, Ryuuji Tsuchiyama, Junguo Xu, Takeshi Harada, Youichi Inoue, Lizhi Su, and Koji Kato, "An active-head slider with a piezoelectric actuator for controlling flying height," IEEE Transactions on Magnetics, Vol. 38, No. 5, 2002.

5. J. Li, M. P. Brenner, J. H. Lang, A. H. Slocum, and R. Struempler, "DRIE-Fabricated curved electrode zipping actuators with low pull in voltage," TRANSDUCERS'03, The 12th International Conference on Solid State Sensors, Actuators and Microsystems, Boston, 2003.

6. M. Shikida, K. Sato, and T. Harada, "Micromachined S-shaped actuator," IEEE Sixth International Symposium on Micro Machine and Human Science, 8:167-172, 1995.

7. M. Yamaguchi, S. Kawamura, K. Minami, and M. Esashi, "Distributed electrostatic micro actuator," IEEE Conference on Microelectromechanical Systems, FL, 18-23, 1993.

8. R. Legtenberg, J. Gilbert, and S. D. Senturia, "Electrostatic curved electrode actuators," Journal of Microelectromechanical Systems, 6:257-265, 1997.

9. R. Jebens, W. Trimmer, and J. Walker, "Microactuators for aligning optical fibers," Sensors and Actuators, 20:6573, 1989.

10. J. Branebjerg and P. Gravesen, "A new electrostatic actuator providing improved stroke length and force," IEEE Conference on Microelectromechanical Systems, Travemunde, Germany, 6-11, 1992. 
11. V. K. Pamula, A. Jog, and R. B. Fair, "Mechanical property measurement of thin-film gold using thermally actuated bimetallic cantilever beams," Proc. Modeling and Simulation of Microsystems, pp. 410-413, 2001.

12. M. T. Hou and R. Chen, "Effect of width on the stress-induced bending of micromachined bilayer cantilevers," Journal of Micromechanics and Microengineering, 13:141-148, 2002.

13. M. W. Judy, Y. Cho, R. T. Howe, and A. P. Pisano, "Self-adjusting microactuators (SAMS)," Proc. IEEE MEMS (MEMS'91), pp. 51-56, 1991.

14. W. H. Chut, M. Mehreganyt, and R. L. Mullen, "Analysis of tip deflection and force of a bimetallic cantilever microactuator," Journal of Micromechanics and Microengineering, 3:4-7, 1993.

15. S. Chowdhury, M. Ahmadi, and W. C. Miller, "A closed-form model for the pull-in voltage of electrostatically actuated cantilever Beam," Journal of Micromechanics and Microengineering, 15:756-763, 2005.

16. http://www.matweb.com 